\title{
On Irrational Points in the Plane
}

\author{
Thomas Beatty, Timothy Jones \\ Florida Gulf Coast University, Fort Myers, Florida, USA \\ Email: tbeatty@fgcu.edu
}

How to cite this paper: Beatty, T. and Jones, T. (2017) On Irrational Points in the Plane. Advances in Pure Mathematics, 7, 299-305.

https://doi.org/10.4236/apm.2017.74016

Received: March 13, 2017

Accepted: April 17, 2017

Published: April 20, 2017

Copyright (c) 2017 by authors and Scientific Research Publishing Inc. This work is licensed under the Creative Commons Attribution International License (CC BY 4.0).

http://creativecommons.org/licenses/by/4.0/

\begin{abstract}
This paper summarizes research intended to develop a pedagogically friendly argument that establishes the fact that $\left(x, e^{x}\right)$ is never a rational point in the plane. A point $(x, y) \in \mathbb{R}^{2}$ is rational if both $x$ and $y$ are rational. Applying a method based on Hurwitz polynomials, the research establishes simple irrationality proofs for nonzero rational powers of $e$ and logarithms of positive rationals (excluding one).
\end{abstract}

\section{Keywords}

Rational Point, Irrationality, Sum of Derivatives, Prime, Divisibility, Hurwitz Polynomial

\section{Introduction}

A certain class of polynomials with integer coefficients displays divisibility properties which can be used to establish irrationality. The purpose of the present research is to exploit this property by evaluating such polynomials at zero and at the base of natural logarithms $e$, which, for the sake of contradiction, is assumed to be rational. Subsequent algebraic manipulations lead to a divisibility argument which forces a contradiction by producing an "integer" strictly between zero and one. This is a well-worn path in demonstrating irrationality, but the specifics for developing such contradictions are highly dependent on the number under consideration. Historically, irrationality proofs for the powers of $e$ were developed for specific integer exponents. Euler [1] proved that both $e$ and $e^{2}$ were irrational in 1737, Liouville showed that $e^{4}$ was irrational in 1840, and Hurwitz [2] proved that $e^{3}$ was likewise irrational in 1891, however this was preceded by a comprehensive answer to all questions of this type when the transcendence of $e$

was established by Hermite [1] [3] in 1873. Observe that if $e^{\frac{m}{n}} \in \mathbb{Q}$, then $\left(e^{\frac{m}{n}}\right)^{n}=e^{m} \in \mathbb{Q}$, hence e solves $x^{m}-e^{m}=0$, contradicting transcendence. The 
method below establishes the same result, namely the irrationality of all non-zero powers of $e$, more simply by avoiding the necessity of showing the transcendence of $e$.

\section{Overview}

Given some $\alpha \in \mathbb{R}$ which is to be shown irrational, construct the following polynomial function with the property that it can be translated from zero to $\alpha$ subject to a controllable error by a simple exponential transformation. Let $G(x) \in \mathbb{Z}[x]$ be the polynomial. Specifically, write $\mathrm{e}^{x} G(0)=G(x)+($ error $)$, where the error term can be dominated by a fixed multiple $\beta$ of the degree of $G(x)$. The trick in making this work is to define $G(x)$ so that it meshes with the pattern by which the Maclaurin expansion for $e^{x}$ restores powers of $x$ to $G(0)$. The exact manner in which this can be done is the subject of a lemma below. The exponential transform is due to Hermite.

The form of $G(x)$ that emerges from the preceding considerations turns out to be the sum of the derivatives of a more basic polynomial, the Hurwitz polynomial of type $p$, defined as $g(x)=x^{p-1}(x-\alpha)^{p}$. The primality of $p$ is not required by the definition, but in the sequel it will be taken to be prime. So define $G(x)=\sum_{n=0}^{\operatorname{deg} g} g^{(n)}(x)$, where the sum runs from 0 to $2 p-1$, since all higher derivatives vanish. Applying Leibnitz' Rule for differentiating products to $g(x)=x^{p-1}(x-\alpha)^{p}$, obtain $g^{(n)}(x)=\sum_{k=0}^{n}\left(\begin{array}{l}n \\ k\end{array}\right)\left(x^{p-1}\right)^{(n-k)}\left((x-\alpha)^{p}\right)^{(k)}$. Hence $G(x)=\sum_{n=0}^{2 p-1}\left[\sum_{k=0}^{n}\left(\begin{array}{l}n \\ k\end{array}\right)\left(x^{p-1}\right)^{(n-k)}\left((x-\alpha)^{p}\right)^{(k)}\right]$, which is truly a cumbersome expression to work with.

There is some computational relief provided by the fact that only $G(x)$ needs to be evaluated at the zeroes of $g(x)$, namely 0 and $\alpha$. Evaluation at these two points automatically eliminates a considerable number of terms.

The principal steps in the argument are:

1) Assume for the sake of contradiction that $\alpha \in \mathbb{Q}^{+}$, say $\alpha=r / s$, where $r, s \in \mathbb{N}$ and $\operatorname{gcd}(r, s)=1$

2) Show $s^{p-1} G(\alpha)$ is an integer divisible by $p$ !

3) Show $s^{p} G(0)$ is an integer divisible by $(p-1)$ ! but not by $p$ !

(iv) Show $\left|\mathrm{e}^{\alpha} G(0)-G(\alpha)\right| \leq \beta(2 p-1)$ recall that the degree of $g(x)$ is $2 p-1$

4) If $\mathrm{e}^{\alpha} \in \mathbb{Q}^{+}$, say $\mathrm{e}^{\alpha}=\frac{u}{v}$, where $u, v \in \mathbb{N}$ and $\operatorname{gcd}(u, v)=1$, then show $|u G(0)-v G(\alpha)| \leq \beta(2 p-1) v$

5) Show $0<s^{p}|u G(0)-v G(\alpha)| \leq s^{p} \beta(2 p-1) v$

6) Show $\lim _{p \rightarrow \infty} \frac{s^{p} \beta(2 p-1) v}{(p-1) !}=0$

7) Establish contradiction by concluding $\frac{s^{p}|u G(0)-v G(\alpha)|}{(p-1) !}$ is a positive 
integer strictly less than one for large $p$

This contradiction yields the main theorem which asserts that $\alpha$ and $\mathrm{e}^{\alpha}$ cannot both be positive rationals. This theorem is used by showing that if one is rational, the other is not. Interesting results flow immediately as corollaries. All positive rational powers of e must be irrational. Natural logarithms of positive rational numbers except 1 cannot be rational.

\section{Main Theorem}

Validation of the technical details of the preceding program follow. It is assumed throughout that $g(x)$ and $G(x)$ are as defined above, $\alpha=\frac{r}{s}$, with $r, s \in \mathbb{N}$ and $\operatorname{gcd}(r, s)=1$, and $p$ is a prime.

Lemma 1: $s^{p-1} G(\alpha)$ is an integer divisible by $p$ !.

Proof: Setting $x=\alpha$ in $G(x)=\sum_{n=0}^{2 p-1}\left[\sum_{k=0}^{n}\left(\begin{array}{l}n \\ k\end{array}\right)\left(x^{p-1}\right)^{(n-k)}\left((x-\alpha)^{p}\right)^{(k)}\right]$ would eliminate all terms except those where $(x-\alpha)^{p}$ has been differentiated $p$ times. The surviving terms would be of the form $p !\left(\begin{array}{c}p+k \\ p\end{array}\right) \frac{(p-1) !}{(p-k-1) !}(\alpha)^{p-k-1}$, corresponding to the situation where $x^{p-1}$ has also been differentiated $k$ times. Hence

$$
G(\alpha)=p ! \sum_{k=0}^{p}\left(\begin{array}{c}
p+k \\
k
\end{array}\right) \frac{(p-1) !}{(p-k-1) !}(\alpha)^{p-k-1}
$$

Simplifying, it is clear that

$$
\begin{aligned}
& G(\alpha)=p ! \sum_{k=0}^{p-1} \frac{(p+k) !}{p ! k !} \frac{(p-1) !}{(p-k-1) !}(\alpha)^{p-k-1}=\sum_{k=0}^{p-1}(p+k) !\left(\begin{array}{c}
p-1 \\
k
\end{array}\right)(\alpha)^{p-k-1} . \\
& \text { Rewriting } \alpha=\frac{r}{s} \text {, evidently } s^{p-1} G(\alpha)=\sum_{k=0}^{p-1}(p+k) !\left(\begin{array}{c}
p-1 \\
k
\end{array}\right) s^{p-1}\left(\frac{r}{s}\right)^{p-k-1},
\end{aligned}
$$
which is an integer divisible by $p$ !, since it divides $(p+k)$ ! for $k \geq 0$ and $\left(\begin{array}{c}p-1 \\ k\end{array}\right)$ is always an integer, establishing the result.

Lemma 2: $s^{p} G(0)$ is an integer divisible by $(p-1)$ ! but not by $p$ ! for sufficiently large p.

Proof: Setting $x=0$ in $G(x)=\sum_{n=0}^{2 p-1}\left[\sum_{k=0}^{n}\left(\begin{array}{l}n \\ k\end{array}\right)\left(x^{p-1}\right)^{(n-k)}\left((x-\alpha)^{p}\right)^{(k)}\right]$ would eliminate all terms except those where $x^{p-1}$ has been differentiated $p-1$ times. The surviving terms would be of the form $(p-1) !\left(\begin{array}{c}p+k-1 \\ k\end{array}\right) \frac{p !}{(p-k) !}(0-\alpha)^{p-k}$, corresponding to the situation where $(x-\alpha)^{p}$ has also been differentiated $k$ times. Hence $G(0)=(p-1) ! \sum_{k=0}^{p}\left(\begin{array}{c}p+k-1 \\ k\end{array}\right) \frac{p !}{(p-k) !}(-\alpha)^{p-k}$. Simplifying further it follows 


$$
\text { that } \begin{aligned}
G(0) & =(p-1) ! \sum_{k=0}^{p}(-1)^{p-k} \frac{(p+k-1) !}{(p-1) ! k !} \frac{p !}{(p-k) !}(\alpha)^{p-k} \text {. Rewriting } \alpha=\frac{r}{s}
\end{aligned}
$$

as above, clearly $s^{p} G(0)=\sum_{k=0}^{p}(-1)^{p-k}(p+k-1) !\left(\begin{array}{l}p \\ k\end{array}\right) s^{p}\left(\frac{r}{s}\right)^{p-k}$. Evidently $(p-1)$ ! divides $s^{p} G(0)$, since it divides $(p+k-1)$ ! for $k \geq 0$ and $\left(\begin{array}{l}p \\ k\end{array}\right)$ is always an integer. On the other hand, for the sake of contradiction, if $p$ ! were to divide $s^{p} G(0)$, then it would divide

$$
\left[s^{p} G(0)-\left[\sum_{k=1}^{p}(-1)^{p-k}(p+k-1) !\left(\begin{array}{l}
p \\
k
\end{array}\right) s^{p}\left(\frac{r}{s}\right)^{p-k}\right]\right]=(-1)^{p}(p-1) !\left(\begin{array}{l}
p \\
0
\end{array}\right)(r)^{p} \text {. }
$$

This is absurd, since it cannot divide the right hand side if $p$ is chosen greater than $r$. Recall $\alpha$, hence $r$, is fixed, but $p$ can be an arbitrary prime. The contradiction establishes that $p$ ! does not divide $s^{p} G(0)$ and the result follows.

Lemma 3.1: Let $\varepsilon_{k}(x)$ denote the infinite series

$$
\frac{x}{k+1}+\frac{x^{2}}{(k+1)(k+2)}+\cdots=\sum_{j=1}^{\infty} \frac{k ! x^{j}}{(k+j) !} \text {. Then } \frac{x^{k}}{k !} \varepsilon_{k}(x)=E_{k}(x),
$$

where

$$
E_{k}(x)=\sum_{j=k+1}^{\infty} \frac{x^{j}}{j !} .
$$

(Note that $E_{k}(x)$ is the series for $\mathrm{e}^{x}$ with the first $k+1$ terms removed.)

Proof: Observe that $\frac{x^{k}}{k !}\left[\sum_{j=1}^{\infty} \frac{k ! x^{j}}{(k+j) !}\right]=\sum_{j=1}^{\infty} \frac{x^{j+k}}{(k+j) !}$, which is equivalent to $\sum_{j=k+1}^{\infty} \frac{x^{j}}{j !}$

Lemma 3.2: Suppose $g(x)=c_{n} x^{n}+\cdots+c_{1} x+c_{0} \in \mathbb{Z}[x]$. Let $G(x)$ be the corresponding sum-of-derivatives polynomial. Then $e^{x} G(0)=G(x)+\sum_{k=0}^{n} c_{k} x^{k} \varepsilon_{k}(x)$, with $\varepsilon_{k}(x)$ as in Lemma 3.1.

Proof: By induction on $\operatorname{deg} g$. If $\operatorname{deg} g=0$, then $g(x)=c_{0}$. There is only the zeroth derivative to sum, so $G(0)=G(x)=c_{0}$. Since $\varepsilon_{0}(x)=\mathrm{e}^{x}-1$., evidently $\mathrm{e}^{x} G(0)=\left(1+\varepsilon_{0}(x)\right) c_{0}=c_{0}+\sum_{k=0}^{0} c_{0} x^{0} \varepsilon_{0}(x)$, establishing the base case.

The induction hypothesis is that for any polynomial $\phi(x)=c_{n-1} x^{n-1}+\cdots+c_{1} x+c_{0}$ of degree $n-1$, we have $\mathrm{e}^{x} \Phi(0)=\Phi(x)+\sum_{k=0}^{n-1} c_{k} x^{k} \varepsilon_{k}(x)$. It is to be shown that for a polynomial of degree $n$, namely $g(x)=\phi(x)+c_{n} x^{n}, \mathrm{e}^{x} G(0)=G(x)+\sum_{k=0}^{n} c_{k} x^{k} \varepsilon_{k}(x)$ it is true that. Now $G(x)=\Phi(x)+\sum_{j=0}^{n}\left(c_{n} x^{n}\right)^{(j)}$, since the only derivatives of $G(x)$ missing from $\Phi(x)$ are those attributable to the leading monomial $c_{n} x^{n}$. Then $G(0)=\Phi(0)+n ! c_{n}$, as all the derivatives except the $n^{\text {th }}$ of $c_{n} x^{n}$ evaluate to 
zero at zero. Forming $\mathrm{e}^{x} G(0)=\mathrm{e}^{x}\left[\Phi(0)+n ! c_{n}\right]$, we have $\mathrm{e}^{x}\left[\Phi(0)+n ! c_{n}\right]=\Phi(x)+\sum_{k=0}^{n-1} c_{k} x^{k} \varepsilon_{k}(x)+\mathrm{e}^{x} n ! c_{n}$ by the induction hypothesis. Observe that $\mathrm{e}^{x} n ! c_{n}=\left(\left[1+\frac{x}{1 !}+\frac{x^{2}}{2 !}+\cdots+\frac{x^{n}}{n !}\right]+\left[\frac{x^{n+1}}{(n+1) !}+\cdots\right]\right) n ! c_{n}$. But $\left[1+\frac{x}{1 !}+\frac{x^{2}}{2 !}+\cdots+\frac{x^{n}}{n !}\right] n ! c_{n}=c_{n} x^{n}+n c_{n} x^{n-1}+n(n-1) c_{n} x^{n-2}+n ! c_{n} x+n ! c_{n}$, which is precisely $\sum_{j=0}^{n}\left(c_{n} x^{n}\right)^{(j)}$. It follows that

$$
\mathrm{e}^{x} G(0)=\left[\Phi(x)+\sum_{j=0}^{n}\left(c_{n} x^{n}\right)^{(j)}\right]+\sum_{k=0}^{n-1} c_{k} x^{k} \varepsilon_{k}(x)+E_{n}(x) n ! c_{n}
$$

where $E_{n}(x)=\sum_{j=n+1}^{\infty} \frac{x^{j}}{j !}$ as in Lemma 3.1. But the term $E_{n}(x) n ! c_{n}=\left(\frac{x^{n}}{n !} \varepsilon_{n}(x)\right) n ! c_{n}=c_{n} x^{n} \varepsilon_{n}(x)$ by Lemma 3.1, hence it can be absorbed and evidently $\sum_{k=0}^{n-1} c_{k} x^{k} \varepsilon_{k}(x)+E_{n}(x) n ! c_{n}=\sum_{k=0}^{n} c_{k} x^{k} \varepsilon_{k}(x)$. Recognizing that $\Phi(x)+\sum_{j=0}^{n}\left(c_{n} x^{n}\right)^{(j)}=G(x)$, it is now clear that $\mathrm{e}^{x} G(0)=G(x)+\sum_{k=0}^{n} c_{k} x^{k} \varepsilon_{k}(x)$, as required.

Lemma 3.3: With the notation of Lemma 3.2, for a given $g(x) \in \mathbb{Z}[x]$ of degree $n$ and fixed $x$, the function $|\varepsilon(n)|=\sum_{k=0}^{n} c_{k} x_{0}^{k} \varepsilon_{k}\left(x_{0}\right)$ is $O(n)$.

Proof: It may be assumed that the fixed $x \neq 0$. From Lemma 3.1 $|\varepsilon(n)|=\left|\sum_{k=0}^{n} c_{k} x^{k} \varepsilon_{k}(x)\right| \leq \sum_{k=0}^{n}\left|c_{k} x^{k} \varepsilon_{k}(x)\right|=\sum_{k=0}^{n}\left|c_{k}\right|\left|x^{k}\right|\left|\varepsilon_{k}(x)\right|$. Note that $\left|E_{k}(x)\right|<\infty$ since $E_{k}(x)$ is dominated by the absolutely convergent series $e^{x}$. Then $\left|\varepsilon_{k}(x)\right|=\frac{k !}{\left|x^{k}\right|}\left|E_{k}(x)\right|$ is bounded, and hence $\sum_{k=0}^{n}\left|c_{k}\right|\left|x^{k}\right|\left|\varepsilon_{k}(x)\right| \leq n \cdot \max _{k \leq n}\left[\left|c_{k}\right|\left|x^{k}\right|\left|\varepsilon_{k}(x)\right|\right]$. It follow that $|\varepsilon(n)| \leq n \beta$, where $\beta=\max _{k \leq n}\left[\left|c_{k}\right|\left|x^{k}\right|\left|\varepsilon_{k}(x)\right|\right]$.

Lemma 3.4: With the notation above, $\left|\mathrm{e}^{\alpha} G(0)-G(\alpha)\right| \leq(2 p-1) \beta$ for some fixed $\beta>0$.

Proof: Lemma 3.2 shows $\mathrm{e}^{x} G(0)-G(x)=\sum_{k=0}^{n} c_{k} x^{k} \varepsilon_{k}(x)$. Hence for $x=\alpha$ it must be that $\left|\mathrm{e}^{\alpha} G(0)-G(\alpha)\right|=\left|\sum_{k=0}^{n} c_{k} \alpha^{k} \varepsilon_{k}(\alpha)\right|=|\varepsilon(p)| \leq(2 p-1) \beta$ by Lemma 3.3, since the degree of the Hurwitz polynomial $g(x)$, which defines $G(x)$ via summation of derivatives, is $2 p-1$. The result is now immediate.

Lemma 4: If $\mathrm{e}^{\alpha} \in \mathbb{Q}^{+}$, say $\mathrm{e}^{\alpha}=\frac{u}{v}$, where $u, v \in \mathbb{N}$ and $\operatorname{gcd}(u, v)=1$, then $|u G(0)-v G(\alpha)| \leq \beta(2 p-1) v$ Moreover, if $\alpha=\frac{r}{s}$ as above, then $0<s^{p}|u G(0)-v G(\alpha)| \leq s^{p} \beta(2 p-1) v$ for sufficiently large $p$.

Proof: From Lemma 3.4, $\left|\mathrm{e}^{\alpha} G(0)-G(\alpha)\right|=\left|\frac{u}{v} G(0)-G(\alpha)\right| \leq(2 p-1) \beta$, so $|u G(0)-v G(\alpha)| \leq \beta(2 p-1) v$. Hence (since $s>0$ ) 
$s^{p}|u G(0)-v G(\alpha)| \leq s^{p} \beta(2 p-1) v$ Note that $s^{p} u G(0)$ is an integer that is not divisible by $p$ ! for sufficiently large $p$ by Lemma 2 , but that $s^{p} v G(\alpha)$ is an integer that is divisible by $p$ ! for any large $p$ by Lemma 1 . Here $p$ can be chosen large enough to not appear in any prime factorization of $s$. It follows that $s^{p}|u G(0)-v G(\alpha)|$ would be a nonzero integer, establishing the claim.

Theorem 1: If $\alpha \in \mathbb{Q}^{+}$then $e^{\alpha} \notin \mathbb{Q}^{+}$.

Proof: To the contrary, suppose $\alpha \in \mathbb{Q}^{+}$and $e^{\alpha} \in \mathbb{Q}^{+}$, then by the lemmas above, $s^{p}|u G(0)-v G(\alpha)|$ would be an integer, and for sufficiently large $p$ would satisfy $0<s^{p}|u G(0)-v G(\alpha)| \leq s^{p} \beta(2 p-1) v$. In the expression on the right hand side, once $\alpha$ is fixed the terms $s, u, v$, and $\beta$ are constant. Claim:

$\lim _{p \rightarrow \infty} \frac{s^{p} \beta(2 p-1) v}{(p-1) !}=0$. The expression can be rewritten $\frac{s^{p-2}}{(p-2) !} \cdot \frac{2 p-1}{p-1} \cdot s^{2} \beta v$ and it is then apparent that the limit must be $2 s^{2} \beta v \cdot \lim _{p \rightarrow \infty} \frac{s^{p-2}}{(p-2) !}$, which is zero due to the factorial dominance over the exponential. So choose a prime $p_{0}$ for which $\frac{s^{p} \beta(2 p-1) v}{(p-1) !}<1$. Now $0<\frac{s^{p_{0}}|u G(0)-v G(\alpha)|}{\left(p_{0}-1\right) !}<1$. But since $\left(p_{0}-1\right)$ ! divides both $G(0)$ and $G(\alpha)$ but does not, by virtue of its selection, divide $s, u$, or $v$, it is clear that $\frac{s^{p_{0}}|u G(0)-v G(\alpha)|}{\left(p_{0}-1\right) !}$ is an integer. Moreover, it is apparently an integer strictly between zero and one, which is absurd. The contradiction establishes the theorem.

\section{Conclusions}

Based on the preceding Theorem 1, the following conclusions are immediate.

Corollary 1: The nonzero rational powers of $e$ are irrational

Proof: For the positive rational powers the statement is true immediately by

Theorem 1. If $\gamma>0$, then $\mathrm{e}^{-\gamma}$ cannot be of the form $\frac{r}{s}$ with $r, s \in \mathbb{N}^{+}$, otherwise $\mathrm{e}^{\gamma}=\frac{s}{r}$, contrary to Theorem 1 .

Corollary 2: The natural logarithm of a positive rational number is irrational

Proof: By the contrapositive of Theorem 1, If $\gamma=\mathrm{e}^{\ln \gamma}$ is rational (necessarily positive), then $\ln \gamma$ is irrational.

Corollary 3: If $\ln \gamma+\ln \delta$ is rational, then either $\gamma \delta$ is irrational or $\gamma \delta=1$

Proof: If $\ln \gamma+\ln \delta$ is a nonzero rational, then $\mathrm{e}^{\ln \gamma+\ln \delta}=\mathrm{e}^{\ln (\gamma \delta)}=\gamma \delta$ is irrational. If $\ln \gamma+\ln \delta=0$, then $\gamma \delta=1$. 


\section{References}

[1] Herstein, I.N. (1975) Topics in Algebra. 2nd Edition, John Wiley \& Sons, New York, 175-178.

[2] Hurwitz, A. (1893) Beweis der Transendenz der Zahl e. Mathematische Annalen, 43, 220-221. https://doi.org/10.1007/BF01443646

[3] Hermite, C. (1873) Sur la fonction exponientielle. Comptes Rendus de l'Academie des Sciences, 77, 18-24.

Submit or recommend next manuscript to SCIRP and we will provide best service for you:

Accepting pre-submission inquiries through Email, Facebook, LinkedIn, Twitter, etc. A wide selection of journals (inclusive of 9 subjects, more than 200 journals)

Providing 24-hour high-quality service

User-friendly online submission system

Fair and swift peer-review system

Efficient typesetting and proofreading procedure

Display of the result of downloads and visits, as well as the number of cited articles

Maximum dissemination of your research work

Submit your manuscript at: http://papersubmission.scirp.org/

Or contact apm@scirp.org 